\title{
Effective viscosity of a suspension of flagellar beating microswimmers: Three-dimensional modeling
}

\author{
Levan Jibuti and Walter Zimmermann ${ }^{*}$ \\ Theoretische Physik I, Universitïßœ Bayreuth, 95440 Bayreuth, Germany \\ Salima Rafaï and Philippe Peylađ \\ LIPhy, Université Grenoble Alpes and CNRS, F-38402 Grenoble, France
}

\begin{abstract}
Micro-organisms usually can swim in their liquid environment by flagellar or ciliary beating. In this numerical work, we analyze the influence of flagellar beating on the orbits of a swimming cell in a shear flow. We also calculate the effect of the flagellar beating on the rheology of a dilute suspension of micro-swimmers. A three-dimensional model is proposed for Chlamydomonas Reinhardtii swimming with a breaststroke-like beating of two anterior flagella modeled by two counter-rotating fore beads. The active swimmer model reveals unusual angular orbits in a linear shear flow. Namely, the swimmer sustains orientations transiently across the flow. Such behavior is a result of the interplay between shear flow and swimmer's periodic beating motion of flagella which exert internal torques on the cell body. This peculiar behavior has some significant consequences on the rheological properties of the suspension. We calculate the Einstein's viscosity of the suspension composed of such isolated modeled microswimmers (dilute case) in a shear flow. We use numerical simulations based on a Rotne-Prager like approximation for hydrodynamic interaction between simplified flagella and the cell body. The results show an increased intrinsic viscosity for active swimmer suspensions in comparison to non-active ones as well as a shear thinning behavior in accordance with previous experimental measurements [Phys. Rev. Lett. 104, 098102 (2010)].
\end{abstract}

PACS numbers: 47.63.Gd, 47.63.mf, 83.10.Pp

\section{INTRODUCTION}

Self-propelled particles and micro-organisms able to swim on a microscopic scale have attracted enormous interest over the last few years [1 5]. Typical examples of microswimmers include biological organisms: microalgae, bacteria, and sperm cells as well as artificial swimmers [2 8]. There are two major categories of swimmers: "pullers" and "pushers." A puller, like for example the micro-alga Chlamydomonas Reinhardtii (CR), has two anterior flagella that pull the fluid toward the cell body along the swimming direction while a pusher (like the bacteria Escherichia coli) pushes the fluid behind the cell body opposite to the swimming direction [1]. Designing controllable microswimmers, capable of detection in vivo and carrying a drug to treat and target localized diseases, is one of the most desired objectives in biophysics. Such a microswimmer should employ special tactics to overcome low Reynolds number constraints for locomotion 9] as well as control their swimming direction or crossstreamline migration [10] in the flow (e.g. Poiseuille flow in blood streams).

At a macro-scale, active fluids are suspensions of selfpropelled micro-organisms (or artificial particles) which by moving, spinning or deforming, significantly alter the macroscopic properties of the fluid [11] like its effective viscosity. After the emergence of theoretical models and simulations of rheological properties [11 17], an increas-

\footnotetext{
* walter.zimmermann@uni-bayreuth.de

$\dagger$ philippe.peyla@univ-grenoble-alpes.fr
}

ing number of experiments has been published on experimental measurements on effective viscosity of active suspensions [18 22]. Sokolov and Aranson 18] measured the viscosity of pusher type bacterial suspension. They report strong decrease of the effective viscosity (up to a factor of 7). Lopez et al. 22] even showed that for semidilute $E$. Coli suspensions the viscous resistance to shear can vanish. The viscosity of planktonic suspension of $C R$ (puller type) has been measured [19] and a significantly higher viscosity was found compared to suspensions containing the same volume fraction of dead cells. Suspensions of living microalgae also show a shear-thinning behavior. In another experiment, Mussler et al. [20] confirmed the previous results using two different geometries (Taylor-Couette and cone-plate) and show that gravity does not play a role in the enhancement of the viscosity unlike suspensions of bottom-heavy Chlamydomonas nivalis 17, 21, 23.

The physical interpretation of this peculiar rheology was first introduced by Hatwalne [12]. It can be summarized as follows. An elongated microswimmer (rod-like bacteria for example), once immersed in a simple shear flow of shear rate $\dot{\gamma}$, spends a long time compared to $\dot{\gamma}^{-1}$ in the extensional direction of the shear flow: this is known as Jeffery orbits 24]. And since each microswimmer is modeled as a permanent force dipole, it increases for pushers or decreases for pullers the off-diagonal stress tensor, respectively, resulting in a decrease or an increase of the effective viscosity. The majority of models relies on this anisotropic orientation distribution of microswimmers in the flow. Such an assumption is natural for the suspensions of pusher type bacteria that have a 
rod-like shape or for gravitactic swimmers that are oriented by gravity [17]. However, it is not applicable to non-gravitactic suspensions of $C R$ which have a spherical body. A sphere has a constant rotation velocity $\Omega=\dot{\gamma} / 2$ in a simple shear flow and no anisotropy prevails. An estimation of the aspect ratio of a puller corresponding to the experimentally measured effective viscosity [19] gives a value of ellipticity of about 7 , far above the value of 1 for a spherical shape! Therefore, the origin of the viscosity enhancement for $C R$ suspensions remains an open question.

Recently, Takatori and Brady [25] introduced a new idea and showed that the diffusion-like motion of microswimmers immersed in a simple shear flow, induces a nonzero off-diagonal shear components in the swim stress tensor. This effect can explain the rheology of a suspension of $C R$. In this work, we also point out a novel physical phenomenon that can also explain the peculiar rheology of $C R$ suspensions. We show that the flagella beating itself, leads to Jeffery-like orbits of the $C R$ in a simple shear flow even if a swimmer's rounded shape is considered. It is shown that because of the coupling between the shear flow and the flagella beating, the swimmer can resist the rotation induced by the shear flow and thus, leads to an increase of effective viscosity. Somehow, our model helps to reconcile preceding theories done with rod-shape swimmers with spherical $C R$ rheology.

The use of a permanent force dipole to describe the swimmer activity 26 28 is reasonable on long time scales. However, in this work, we show that even if the flow characteristic time $\dot{\gamma}^{-1}$ is much larger than the beating period $T_{b}$, the flagellar breaststroke gives rise to peculiar Jeffery orbits of the swimmer and thus, affects the rheology of the dilute suspension composed of these swimmers.

In this work, we propose and investigate a threebead model for a swimming Chlamydomonas Reinhardtii, where the beads are connected by a frictionless scaffold. Our 3d model shares similarities with the twodimensional models for $C R$, described in Ref. [29] and in Ref. [30]. While in both works three equal-sized spheres are used, the beads representing the flagella are of different size in Ref. [31]. The flagellar beads in these $2 \mathrm{~d}$ models move on circular orbits. Here, we investigate within our $3 \mathrm{~d}$ model the effect of the flagellar beating on a $C R$ motility in a simple shear flow and its consequence on the rheology of a dilute suspension of $C R$. The model is presented in section $I I$, the calculation of Einstein's viscosity is given in section $I I I$ and our results are given in section $I V$.

\section{MODEL FOR BIFLAGELLATE ALGAE}

A three-dimensional swimmer-model of a biflagellate alga Chlamydomonas Reinhardtii is described in this section. The body of the $C R$ alga is described by a sphere of radius $R$. This is linked to two smaller satellite beads of radius $r$ mimicking the two flagella of the alga, as indicated in Fig. 1, Each of the the left $(L)$ and right $(R)$ flagellar beads is connected to the body by three springs $S_{m}^{L, R}, S_{s a}^{L, R}, S_{s b}^{L, R}$ (superscripts $L$ or $R$ for left and right bead, respectively). The main springs $S_{m}^{L, R}$ are chosen more rigid than the two supporting springs $S_{s a}^{L, R}$ and $S_{s b}^{L, R}$ (see table I), which help to maintain both satellite beads into the $(\hat{\mathbf{m}}, \hat{\mathbf{n}})$ plane, cf. Fig. [1. This anchoring of each satellite bead also allows the application of torques to the central bead, similarly to the flagella on the body of a $C R$. In the case of an active swimmer-model, the equilibrium lengths of the springs are time dependent, in order to mimic the characteristic breaststroke-like swimming motion of a $C R$. For a dead swimmer equilibrium lengths become time-independent during a tumbling motion in a shear flow.

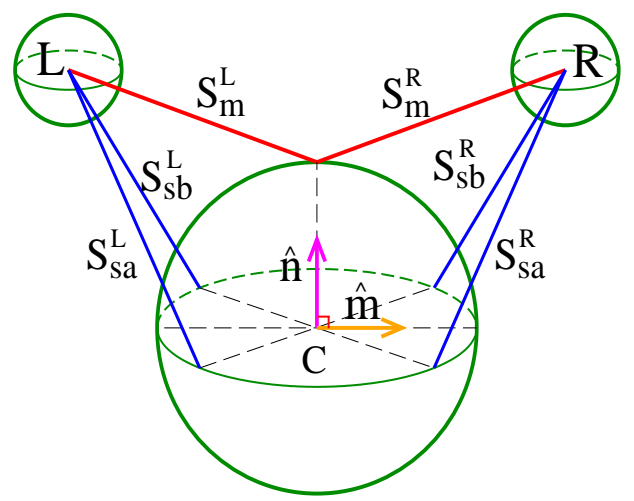

FIG. 1. The active swimmer-model consists of three beads where the left $(L)$ and the right $(R)$ satellite bead of radius $R^{\prime}$ are connected to the body-bead of radius $R$ and centered in $C$ by frictionless Hookean springs, $S_{s a, s b, m}^{L}$ or $S_{s a, s b, m}^{R}$, respectively. $R / R^{\prime}=3$ and the equilibrium length of the springs can be chosen time-dependent or stationary. The unit vector $\hat{\mathbf{n}}$ defines the mean swimming direction and defines together with the unit vector $\hat{\mathbf{m}}$ the mean flagellar beating plane.

The two perpendicular unit vectors, $\hat{\mathbf{n}}$ and $\hat{\mathbf{m}}$, span the mean "flagella plane" of the orbiting satellite beads. $\hat{\mathbf{n}}$ points from the center of the body to the common anchoring point of the two main springs $S_{m}^{L, R}$ (Fig. 1) and describes the mean swimming direction of a swimmer. The other unit vector $\hat{\mathbf{m}}$ points from the center of the body to the midpoint of the line between the two anchoring points of the supporting springs, $S_{s a}^{R}$ and $S_{s b}^{R}$. The four anchoring points of the supporting springs build a square in the equatorial plane of the central bead with diagonals of length $2 R$ and with the vector $\hat{\mathbf{n}}$ normal to this square.

A swimming motion is induced when the length of the Hookean springs, $\ell_{i}(t)$, is periodically modulated with a frequency $\omega=2 \pi / T_{b}: \ell_{i}(t)=\ell_{i, 0}+A_{i} \cos \left(\omega t+\varphi_{i}\right)$. The spring length in the relaxed state is $\ell_{i, 0}$, the modulation amplitude is $A_{i}$ and the phase $\varphi_{i}$. Each satellite bead imposes via the springs, either during a swimming motion $\left(A_{i} \neq 0\right)$ or when the passive swimmer $\left(A_{i}=0\right)$ tumbles 


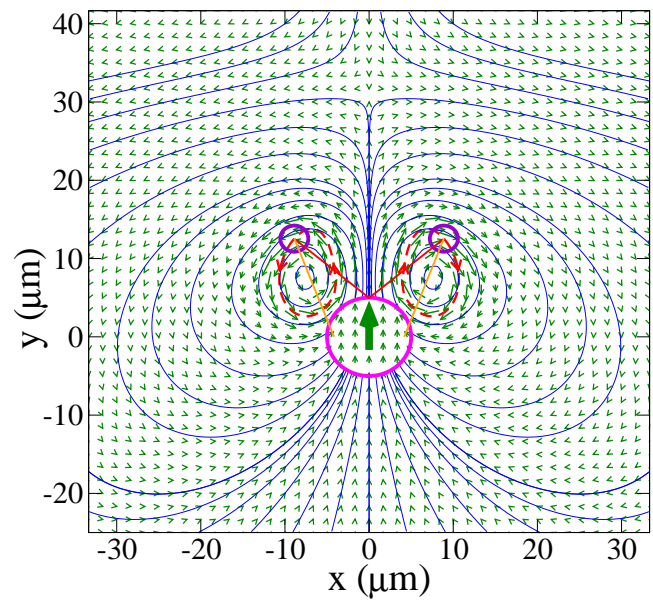

FIG. 2. The velocity field and streamlines around the model swimmer in a fluid at rest (in the lab reference frame) and averaged over one period of the flagellar beating. The arrow inside the main sphere indicates the motion of the swimmer. The closed dashed curves represent the orbits of each satellite bead with respect to the central bead. The swimmer moves back and forth during one period but in average it moves forward with a velocity $v \sim 54 \mu \mathrm{m} / \mathrm{s}$ obtained for chosen parameters (see text and table I). The flow lines as well as the velocity field compare very well with the experimental results of Drescher et al. [27].

in a shear flow, via the springs forces and torques to the central bead.

The 25 spring parameters $A_{i}, \ell_{i, 0}, \varphi_{i}$ and the spring constant $k_{i}$ for each of the six springs as well as the frequency $\omega$ define the characteristics of the swimmer. This number of parameters can be drastically reduced by assuming symmetries of the swimmer. One is the mirror symmetry with respect to a plane perpendicular to $\hat{\mathbf{m}}$, i.e. parameters of left and right springs are identical. We also assume that the swimmer is not rotating around its mean swimming direction $\hat{\mathbf{n}}$, i.e. we can choose identical spring constants for $S_{s a}^{L, R}$ and $S_{s b}^{L, R}$. Only the differences between the phases of the oscillating main and supporting springs on each side are important, namely $\delta \varphi_{a, b}^{L, R}=\varphi_{s a, s b}^{L, R}-\varphi_{m}^{L, R}$ with $\delta \varphi_{a}^{L, R}=\delta \varphi_{b}^{L, R}=\delta \varphi$. Hence, we are left with eight independent parameters: $\ell_{m 0}, \ell_{s 0}$, $A_{m}, A_{s}, \delta \varphi, \omega, k_{m}$ and $k_{s}$.

The $C R$ alga has approximately a spherical shape with diameter $2 R=10 \mu m$ and for particles of this size the Brownian dynamics plays a minor role. Considering the kinematic viscosity for water $\nu \approx 10^{-6} \mathrm{~m}^{2} \mathrm{~s}^{-1}$ the Reynolds number is rather low: $R e=v R / \nu \sim 2.5 \times 10^{-4}$ for a typical swimmer velocity $v \approx 50 \mu \mathrm{m} / \mathrm{s}$. Therefore, inertial forces can be neglected and the fluid flow can be described by the Stokes equation [32]. The particle dynamics and hydrodynamic particle-particle interactions can be described through a reflection method similar to the one used in the Rotne-Prager approximation 32 34].

Let us summarize our mathematical treatment below. Consider the $i^{t h}$ sphere $(i=1 . .3)$ with radius $R_{i}$. The particle is submitted to an external force $\mathbf{F}_{i}$ and a torque $\mathbf{T}_{i}$ with no slip boundary condition. In our model, there is no net force or net torque exerted on the swimmer. However, oscillating springs can apply equal and opposite forces on beads that are aligned along the springs. Because of the design of our model, each spring can exert a torque only on the central bead, whereby the total torque exerted by the six springs on the central bead vanishes in a quiescent fluid.

At zeroth order, $\mathbf{v}_{0, i}=\mathbf{F}_{i} /\left(6 \pi \eta R_{i}\right)$ and $\boldsymbol{\Omega}_{0, i}=$ $\mathbf{T}_{i} /\left(8 \pi \eta R_{i}\right)$ are respectively the translational velocity and the rotational frequency of the $i^{t h}$ particle. When the model swimmer in Fig. (1) is placed in a quiescent fluid, the flow field at zeroth order $\mathbf{u}_{0}$ expressed in $\mathbf{r}$ is:

$$
\mathbf{u}_{0}(\mathbf{r})=\sum_{i=1}^{3} \mathbf{u}_{0, i}\left(\boldsymbol{\rho}_{i}\right)=\sum_{i=1}^{3}\left[\mathbf{V}_{0, i}^{t}\left(\boldsymbol{\rho}_{i}\right)+\mathbf{V}_{0, i}^{r}\left(\boldsymbol{\rho}_{i}\right)\right]
$$

where $\boldsymbol{\rho}_{i}$ is the position vector pointing from the $i^{\text {th }}$ sphere's center to a given point $\mathbf{r}$ in the fluid: $\boldsymbol{\rho}_{i}=\mathbf{r}-\mathbf{r}_{i}$. The summation is carried out on the three interacting spherical beads of the model swimmer. The result averaged over one period of beating is represented in Fig. 2 , i.e. $2 \pi / \omega$. Here, $\mathbf{V}_{0, i}^{t}$ and $\mathbf{V}_{0, i}^{r}$ are respectively the translational and rotational parts of the flow field created by the moving and rotating $i^{\text {th }}$ sphere with radius $R_{i}$, velocity $\mathbf{v}_{0, i}$ and angular velocity $\boldsymbol{\Omega}_{0, i}[41]$ :

$$
\begin{aligned}
\mathbf{V}_{0, i}^{t}\left(\boldsymbol{\rho}_{i}\right)= & \mathbf{v}_{0, i} \frac{3 R_{i}}{4 \rho_{i}}\left(1+\frac{R_{i}^{2}}{3 \rho_{i}^{2}}\right)+ \\
& +\boldsymbol{\rho}_{i}\left(\mathbf{v}_{0, i} \cdot \boldsymbol{\rho}_{i}\right) \frac{3 R_{i}}{4 \rho_{i}^{3}}\left(1-\frac{R_{i}^{2}}{\rho_{i}^{2}}\right), \\
\mathbf{V}_{0, i}^{r}\left(\boldsymbol{\rho}_{i}\right)= & \left(\frac{R_{i}}{\rho_{i}}\right)^{3} \boldsymbol{\Omega}_{0, i} \times \boldsymbol{\rho}_{i} .
\end{aligned}
$$

This, in turn, influences the motion of the $j^{\text {th }}(j=1 . .3)$ particle centered in $r_{j}$ which is calculated via the Faxén laws [32] at first order:

$$
\begin{aligned}
& \mathbf{v}_{j, 1}=\frac{\mathbf{F}_{j}}{6 \pi \eta R_{j}}+\left.\sum_{i \neq j}^{3}\left(1+\frac{R_{j}^{2}}{6} \triangle\right) \mathbf{u}_{0, j}\right|_{r_{j}}, \\
& \boldsymbol{\Omega}_{j, 1}=\frac{\mathbf{T}_{j}}{8 \pi \eta R_{j}}+\frac{1}{2} \sum_{i \neq j}^{3} \nabla \times\left.\mathbf{u}_{0, j}\right|_{r_{j}} .
\end{aligned}
$$

The quantities $\mathbf{u}_{0, i}, \triangle \mathbf{u}_{0, i}$ and $\boldsymbol{\nabla} \times \mathbf{u}_{0, i}$ are estimated at the center of the $j^{t h}$ particle. When the model swimmer is immersed in a shear flow [44], the shear induced velocity $\mathbf{V}_{i}^{*}\left(\boldsymbol{\rho}_{i}\right)$ must be added to the flow field $\mathbf{u}_{0, i}\left(\boldsymbol{\rho}_{i}\right)$ for each spherical bead $i$ :

$$
\begin{aligned}
& V_{x, i}^{*}\left(\boldsymbol{\rho}_{i}\right)=\dot{\gamma}\left[y_{i}-\frac{y_{i} R_{i}^{5}}{2 \rho_{i}^{5}}-\frac{5}{2} \frac{x_{i}^{2} y_{i}}{R_{i}^{2}}\left(\frac{R_{i}^{5}}{\rho_{i}^{5}}-\frac{R_{i}^{7}}{\rho_{i}^{7}}\right)\right], \\
& V_{y, i}^{*}\left(\boldsymbol{\rho}_{i}\right)=\dot{\gamma}\left[-\frac{x_{i} R_{i}^{5}}{2 \rho_{i}^{5}}-\frac{5}{2} \frac{x_{i} y_{i}^{2}}{R_{i}^{2}}\left(\frac{R_{i}^{5}}{\rho_{i}^{5}}-\frac{R_{i}^{7}}{\rho_{i}^{7}}\right)\right], \\
& V_{z, i}^{*}\left(\boldsymbol{\rho}_{i}\right)=-\dot{\gamma} \frac{5}{2} \frac{x_{i} y_{i} z_{i}}{R_{i}^{2}}\left(\frac{R_{i}^{5}}{\rho_{i}^{5}}-\frac{R_{i}^{7}}{\rho_{i}^{7}}\right) .
\end{aligned}
$$


Here $\rho_{i}=\sqrt{x_{i}^{2}+y_{i}^{2}+z_{i}^{2}}, x O y$ is the shear plane and $\dot{\gamma}$ is the imposed shear rate. Using the approximation of the Faxén theorems and keeping the first iteration step in the reflection of hydrodynamic interactions leads to a method valid up to order of $(R / r)^{3}$ [37].

For a given set of parameters, including the oscillation amplitudes $A_{m}$, the two satellites move in general on elliptical orbits as indicated in Fig. 2. The left (resp. right) bead is orbiting counter-clockwise (resp. clockwise). This bead motion mimics the motion of the flagella of $C R$ [35]: The model swimmer moves forward (along $\hat{\mathbf{n}}$ ) during a power stroke (i.e. when satellite beads move in the direction of the body) and moves backward during a recovery stroke (i.e. when satellite beads move in the opposite direction). This resembles very much the experimentally observed vacillating swimming motion of $C R$ [35]. Fig. 2 shows besides the orbits of the satellite beads the velocity field and the streamlines averaged over one period of the motion of the satellite beads.

The parameters in table 1 are chosen in such a way that they reproduce rather closely the swimming characteristics of a $C R$. For instance, the swimming velocity of the model (averaged over the orbiting period of the satellite beads $\left.T_{b}=2 \pi / \omega=1 / 50 \mathrm{~s}\right)$ is $v=540 R \omega \sim 54 \mu \mathrm{m} / \mathrm{s}$ considering a radius $R=5 \mu \mathrm{m}$. Note that if spring parameters are chosen such that $k_{m} / k_{s}<1$ and $\delta \varphi \sim \pi$, then the motion of the satellite beads along their orbits is reversed, and the swimmer would move backward along the $-\hat{\mathbf{n}}$ direction like a pusher.

TABLE I. Parameter values of our model.

\begin{tabular}{|c||c|c|c|c|c|c|c|c|}
\hline & $\frac{R}{r}$ & $\frac{k_{s}}{k_{m}}$ & $\frac{\ell_{m} 0}{R}$ & $\frac{\ell_{s}}{R}$ & $\frac{A_{m}}{R}$ & $\frac{A_{s}}{R}$ & $\frac{\omega}{2 \pi}$ & $\delta \varphi$ \\
\hline \hline active & 3 & $4 / 7$ & 1.9 & 1.8 & 1.3 & 0.8 & $50 H z$ & $\pi / 3$ \\
\hline inactive & 3 & 0 & 1.9 & - & - & - & - & - \\
\hline
\end{tabular}

For any asymmetry between the spring parameters of the left and right satellite bead, swimming becomes less efficient compared to the case of a symmetric and synchronous motion of the satellite beads. Moreover, the mean swimming direction is not stationary anymore and moves for instance on curved trajectories or leads to other complex swimming trajectories as reported for $C R[36]$.

The model microswimmer with oscillating harmonic springs imitates the swimming of a $C R$ and is referred to as "active swimmer". We call it "inactive swimmer" or dead swimmer when the supporting springs $S_{s a}^{L, R}$ and $S_{s b}^{L, R}$ are removed and the modulation amplitudes $A_{m}$ vanish. Then, the satellite beads are very flexible connected to the body, i. e. they are allowed to move freely in the vicinity of the central bead due to an external flow, similar to the flagella of a dead $C R$.

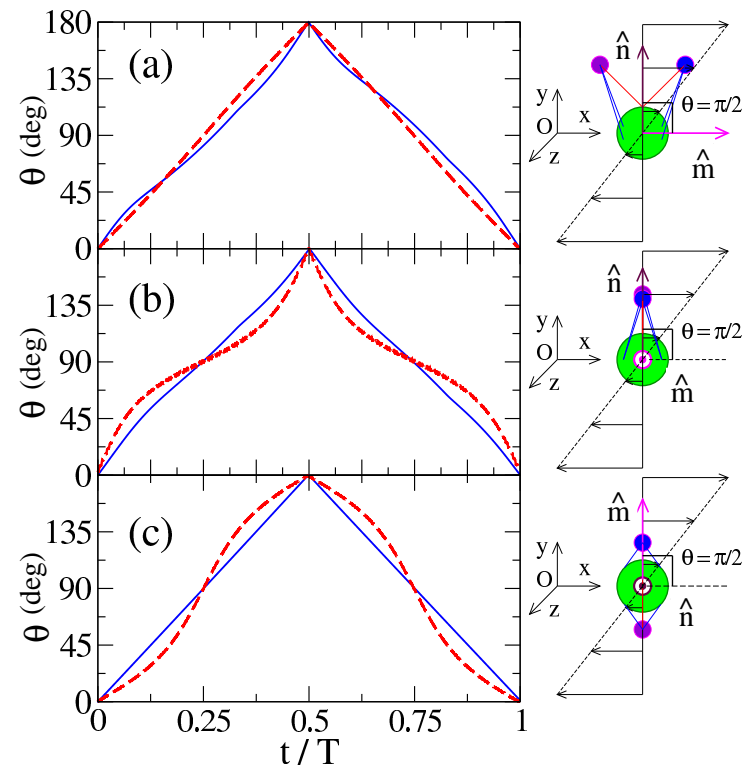

FIG. 3. Temporal orientation angles of active (dashed lines) or inactive microswimmers (solid line) in a linear shear flow for three different swimmer orientations. (a) $\hat{\mathbf{n}}$ (swimming direction) and $\hat{\mathbf{m}}$ are both parallel to the shear ( $x y)$ plane. (b) $\hat{\mathbf{n}}$ parallel to the $x y$ plane and $\hat{\mathbf{m}} \| \hat{\mathbf{z}}$. (c) $\hat{\mathbf{m}} \|$ to the shear plane and $\hat{\mathbf{n}} \| \hat{\mathbf{z}}$. In parts (a) and (b) $\theta$ is the angle between $\hat{\mathbf{n}}$ and $\hat{\mathbf{x}}$, while in part (c) $\theta$ is the angle between $\hat{\mathbf{m}}$ and $\hat{\mathbf{x}}$. The time dependence is plotted in units of the shear rate dependent tumbling period $T$, which is different for each curve [see table III.

\section{INTRINSIC VISCOSITY OF A DILUTE SUSPENSION OF MICROSWIMMERS.}

In this section, we consider a single microswimmer in a linear shear flow $\mathbf{v}=(\dot{\gamma} y, 0,0)$ with the shear rate $\dot{\gamma}$ and its contribution to the effective shear viscosity. Hydrodynamic interactions among swimmers are neglected, which corresponds to a dilute swimmer suspension.

For a dilute suspension of rigid particles the effective viscosity depends linearly on the volume fraction $\phi$ occupied by the particles:

$$
\eta_{\text {eff }}=\eta(1+\alpha \phi)
$$

$\eta$ is the shear viscosity of the solvent and the dimensionless coefficient $\alpha$ is the intrinsic viscosity and $\phi=V_{0} / V$ where $V_{0}=4 / 3 \pi\left(R^{3}+2 R^{\prime 3}\right)$ is the volume of the swimmer and $V$ is the total volume of the suspension (swimmer and water). The quantity $\alpha$ is known as the Einstein's intrinsic viscosity [38, 39]. In general, $\alpha$ is defined as

$$
\alpha=\lim _{\phi \rightarrow 0} \frac{\eta_{e f f}-\eta}{\eta \phi} .
$$

Following the Batchelor method and neglecting inertia, the average stresslet $S_{i k}^{(p)}$ induced by a particle in the fluid is as follows [40, 41]:

$$
S_{i k}^{(p)}=\oint_{\mathcal{A}}\left\{\sigma_{i j} x_{k} \mathcal{N}_{j}-\eta\left(v_{i} \mathcal{N}_{k}+v_{k} \mathcal{N}_{i}\right)\right\} d A
$$


TABLE II. Intrinsic viscosity for different swimmer orientations.

\begin{tabular}{|c||c|c|}
\hline & $\begin{array}{c}\alpha \\
\text { inactive }\end{array}$ & $\begin{array}{c}\alpha \\
\text { active }\end{array}$ \\
\hline \hline$\hat{\mathbf{n}} \angle x y ; \hat{\mathbf{m}} \angle x y$ & 3.0 & 5.7 \\
\hline$\hat{\mathbf{n}} \angle x y ; \hat{\mathbf{m}} \| z$ & 2.9 & 4.8 \\
\hline$\hat{\mathbf{m}} \angle x y ; \hat{\mathbf{n}} \| z$ & 2.5 & 3.5 \\
\hline all configurations & 2.7 & 4.4 \\
\hline experimental [19, 20] & $2.5 \pm 0.1$ & $4.5 \pm 0.2$ \\
\hline
\end{tabular}

TABLE III. Period of swimmer tumbling. Note that the tumbling period for a single sphere depends on the shear rate $\dot{\gamma}$ : $T=2 \pi /|\Omega|=4 \pi / \dot{\gamma} \simeq 126 T_{b}$.

\begin{tabular}{|c||c|c|}
\hline & $\begin{array}{c}T / T_{b} \\
\text { inactive }\end{array}$ & $\begin{array}{c}T / T_{b} \\
\text { active }\end{array}$ \\
\hline \hline$\hat{\mathbf{n}} \angle x y ; \hat{\mathbf{m}} \angle x y$ & 126 & 146 \\
\hline$\hat{\mathbf{n}} \angle x y ; \hat{\mathbf{m}} \| z$ & 145 & 166 \\
\hline$\hat{\mathbf{m}} \angle x y ; \hat{\mathbf{n}} \| z$ & 127 & 150 \\
\hline
\end{tabular}

$\hat{\mathcal{N}}$ is the unit outward vector normal to the surface $\mathcal{A}$ encompassing a volume $V$. Any closed surface $\mathcal{A}$ can be selected for integration as long as it contains the swimmer. The average total shear stress in a volume $V$ containing a single swimmer is $\Sigma_{x y}=\eta \dot{\gamma}+S_{x y}^{(p)} / V=\eta \dot{\gamma}+S_{x y}^{(p)} \phi / V_{0}$, $V_{0}$ being the volume of the swimmer. Note that in the absence of an external torque imposed on the swimmer $\boldsymbol{S}^{(p)}$ is symmetric and only the deviatoric part $\Sigma_{x y}$ of the stress is significant [40]. The effective viscosity is then calculated such as $\eta_{e f f}=\Sigma_{x y} / \dot{\gamma}$ which gives via Eq. (7):

$$
\alpha=\frac{1}{\eta \dot{\gamma} V_{0}} S_{x y}^{(p)} .
$$

In the case of a single rigid sphere the exact velocity and pressure fields around the sphere are known analytically and the calculation of $\alpha$ gives the well known value: $\alpha=$ $5 / 2$ [38, 39].

A significant enhancement of the effective viscosity for a suspension of living $C R$ is reported from experiments [19, 20]. Hydrodynamic interactions among swimmers as well as confinement effects [42] may play an important role for concentrated suspensions. Here, our analysis based on an isolated single swimmer does not include such effects and is limited to dilute suspensions $(\phi<0.01)$, which is usually the case for a natural planktonic suspension. We compare the numerically calculated intrinsic viscosity, $\alpha$, for dilute suspensions to the experimentally observed value $\alpha_{\exp }$ [19], estimated by a fit according to the Krieger and Dougherty's law [43].

The temporal modulation of the equilibrium length of the springs and the exposition of the swimmer to the linear shear flow leads to a complex dynamics of the three beads and therefore to a time-dependent intrinsic viscosity $\alpha$. The flow field around the swimmer is a superposition of the imposed linear shear flow and flow perturbations caused by the bead dynamics. Then the numerical surface integration in Eq. (9) provides a value for $\alpha(t)$ [through Eq. (10)] for each temporal configuration of the microswimmer, i.e. at each time $t$.

The calculation of the intrinsic viscosity $\alpha(t)$ at a chosen time $t$ via Eq. (10) strongly depends on the relative positions of satellite beads on their orbits. It also depends on the orientation of the microswimmer and of its flagella plane $(\hat{\mathbf{n}}, \hat{\mathbf{m}})$ with respect to the flow direction $(\hat{\mathbf{x}})$ and the shear is the plane (xy-plane). Therefore, $\alpha$ is averaged over the tumbling period $T$ of the microswimmer in a shear flow (see table II). Note that one has usually $T \gg T_{b}=2 \pi / \omega$ (c.f. table III), where $T$ is of the same order of magnitude than the period of the rotational part of the shear flow: $2 \pi / \Lambda=4 \pi / \dot{\gamma}$ (with $\Lambda \sim \dot{\gamma} / 2$ ). The tumbling period of swimmers depends also on the swimmer's orientations [see Table III. In dilute suspensions, we assume that any orientation of a swimmer is equally probable, which may not be true for gravitactic microswimmers - not considered here. Therefore, before comparing our numerical results for $\alpha$ with experimental measurements, we take the ensemble average of different realizations of the swimmer's orientations with respect to the flow direction and the shear plane. Results are discussed in the following section.

\section{RESULTS AND DISCUSSION}

The intrinsic viscosity of the model microswimmer is evaluated by averaging over all swimmer orientations and for the shear rate $\dot{\gamma}=5 s^{-1}$, which corresponds to the experimental value [19, 20]. For an active swimmer suspension, we obtain $\alpha=4.4$ and for an inactive swimmer suspension $\alpha=2.7$. The intrinsic viscosity, of the active swimmer suspension is consistent with the experimentally measured viscosity $\alpha_{\exp }=4.5 \pm 0.2$ in Ref. [19] and its $\alpha_{\text {exp }}=4.5 \pm 0.17$ in Ref. [20]. For the suspension of inactive swimmers the numerically calculated intrinsic viscosity is also comparable to the experimental value $\alpha_{\text {exp }}=2.5 \pm 0.1$ [19].

In our averaging, all orientations of the swimmer in the shear flow are possible and each of them corresponds to a different Jeffery orbit. Next, we consider some specific orientations of active and inactive swimmers with respect to the shear flow, for exploring the origin of the viscosity enhancement. In the first configuration in Fig. 3(a) swimmers tumble with $\hat{\mathbf{m}}$ and $\hat{\mathbf{n}}$ in the shear plane $(x y)$. In this case, the intrinsic viscosity for an active swimmer is $\alpha=5.7$ [see table 1 ] and for an inactive swimmer we obtain a smaller value $\alpha=3.0$. The angular velocity of an active swimmer is surprisingly uniform, as indicated by the dashed curve in Fig. 3(a). In addition, the tumbling period $T \simeq 146 T_{b}$ of an active swimmer is considerably larger than for an inactive one, $T \simeq 126 T_{b}$, which is equal to the tumbling period of a sphere of the same radius, $T=2 \pi /|\Lambda|=4 \pi / \dot{\gamma} \simeq 126 T_{b}$.

A more unexpected orbit has been found for the active swimmer with $\hat{\mathbf{n}}$ in the shear plane and $\hat{\mathbf{m}}$ parallel to $\hat{\mathbf{z}}$, cf. Fig. 3(b). The active swimmer rotates slower when 


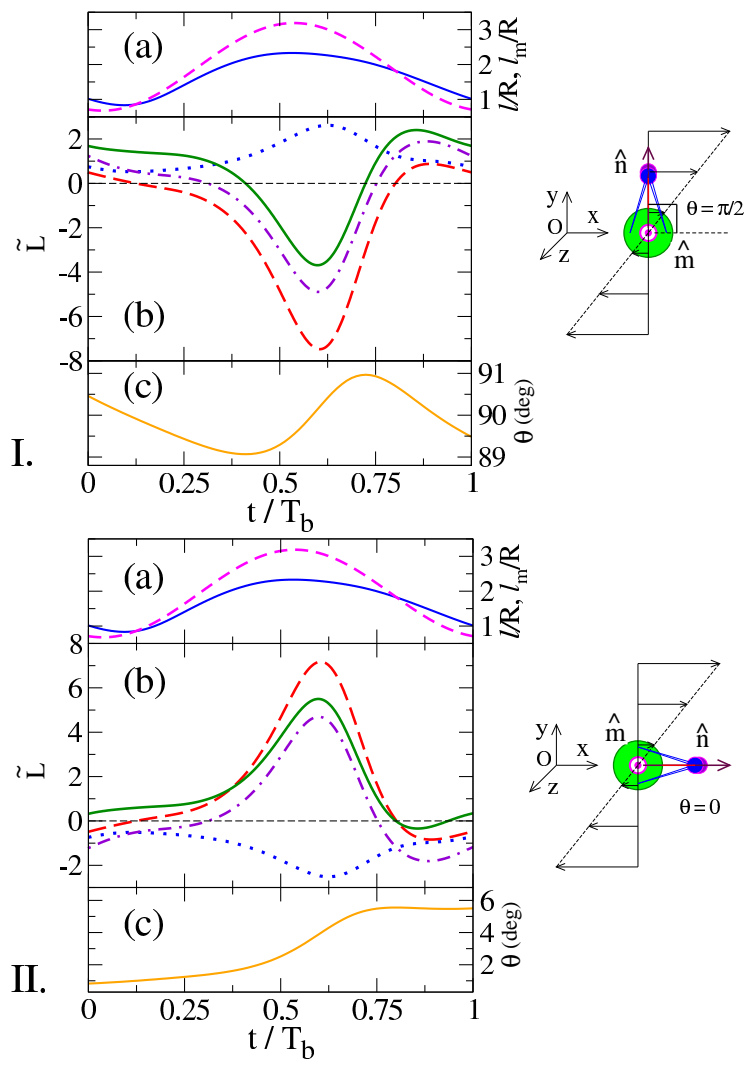

FIG. 4. The top part $\mathbf{I}$ is for the swimming direction perpendicular to the flow direction, i. e. $\hat{\mathbf{n}} \| y$, and $\hat{\mathbf{m}} \| z$. The bottom part II is for the swimming direction parallel to the flow direction, i. e. $\hat{\mathbf{n}} \| x$, and $\hat{\mathbf{m}} \| z$. In I and in II part (a) shows the time-dependence of the actual length $l(t)$ of the main springs in units of $R$ (solid curve) and the time-dependent equilibrium length $l_{m}(t)$ of the main springs (dashed curve) during one period $T_{b}$ of the satellite bead motion. Part (b) shows in I and in II the dimensionless torque originating from the main springs (dashed curve), the supporting springs (dotted curve) and all spring together (dashed-dotted curve). Solid curve shows the total torque exerted by the satellite beads on the body. (c) shows the orientation angle $\theta(t)$ of the swimmer in the shear flow.

$\hat{\mathbf{n}}$ is roughly parallel to $\pm y(\theta \sim \pi / 2)$ and rotates faster with $\hat{\mathbf{n}}$ nearly parallel to the flow, i.e. $\theta \sim 0$ or $\theta \sim \pi$ [see dashed curve on Fig. 3(b)]. This is in contrast to the behavior of an elongated object in a shear flow. For the configuration in Fig. 3(b) the tumbling period and the intrinsic viscosity for inactive swimmer, $T \simeq 145 T_{b}$ and $\alpha=2.9$, are again considerably smaller than for an active swimmer with $T \simeq 166 T_{b}$ and $\alpha=4.8$.

We find the same trend for the third configuration in Fig. [3(c), where we consider a swimmer where $\hat{\mathbf{m}}$ is in the shear plane and $\hat{\mathbf{n}}$ parallel to $\hat{\mathbf{z}}$. Here, the active swimmer is tumbling on usual Jeffery-like orbits [see Fig. 3(c)]. Again the tumbling period and the intrinsic viscosity of an inactive swimmer are respectively $T \simeq 127 T_{b}$ and $\alpha=$ 2.5, and are smaller than for a active swimmer: $T \simeq$ $150 T_{b}$ and $\alpha=3.5$.

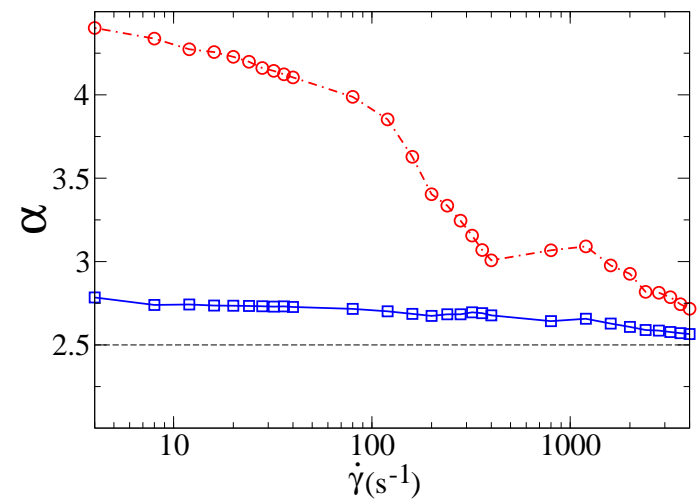

FIG. 5. The intrinsic viscosity as a function of the shear rate. Solid line (squares) corresponds to inactive swimmers and the dashed line (circles) to the active swimmers.

In the case of any other arbitrary orientation of a swimmer with respect to the shear plane, the effective viscosity smoothly changes between the corresponding configurations discussed before. The enhancement of the intrinsic viscosity and the reduction of the angular velocity for active swimmers in comparison to inactive swimmers have their origin on the one hand in the different torques active and passive swimmers experience during their tumbling dynamics in a shear flow. On the other hand, the satellites are kept by the springs at a certain mean distance from the main body, which enhances the effective diameter of the active swimmer compared to the inactive swimmer. Indeed, for an inactive swimmer, satellites are fixed only by one spring and are therefore very flexible like the flagella of a dead $C R$.

The satellite beads anchored to the body by springs with time-dependent equilibrium lengths in combination with the shear flow exert a torque on the cell body. This causes changes of the angular velocity of an active swimmer, compared to the one of a rigid sphere without satellites. When a swimmer with mirror symmetry swims without an external flow, each spring exerts a torque on the body, but the sum of the torques exerted by all springs is zero and swimmer moves along spontaneous (initial) orientations. In Figure 4, two configurations are considered with the flagella plane perpendicular to the shear plane, and the swimming direction either perpendicular (I) or parallel (II) to the flow direction. We define the dimensionless torque, $\widetilde{L}=-L /\left(4 \pi \eta R^{3} \dot{\gamma}\right)$, imposed by the flagella on the swimmer's body. $L$ is the torque applied on the body by the satellites via springs and $L_{s}=-4 \pi \eta R^{3} \dot{\gamma}$ is the torque imposed by the shear flow on a spherical body of the same radius but without satellite beads. For $\widetilde{L}<0$ the torque experienced by the swimmer is opposite to the torque exerted by the shear flow.

Figure 4I shows the swimmer dynamics when flagella plane and swimming direction are both perpendicular to the flow direction. Figure $4 \mathrm{I}(\mathrm{a})$ shows the time dependence of the lengths of the main springs, $\ell(t)$ (solid 
curve), and the imposed time-dependence of the equilibrium length of the main springs, $\ell_{m}(t)$ (dashed curve), during an orbital period $T_{b}$ of the satellite beads. When the main springs are compressed $\left(\ell<\ell_{m}\right)$, the torque exerted by the main springs to the body is opposite to the torque applied by the shear flow. Meanwhile, the supporting springs exerts a torque on the body which have the same sign as the torque applied by the shear flow. However, the total torque exerted by all springs on the body is opposite to the one imposed by the shear flow. Altogether, the angular velocity of the swimmer is reduced, and the swimmer stays longer along the swimming direction perpendicular to the flow, in comparison to a spherical particle of the same radius as the body bead Fig. 3 I(b) [see also table III]. It is remarkable that such a behavior is opposite to the usual Jeffery orbit of a passive elongated object in a shear flow [24].

In contrast, when the flagella plane is still perpendicular to the flow but with the swimming direction parallel to the flow as in Fig. 4HII, the swimmer behaves in an opposite way. The total torque exerted by all springs on the swimmer amplifies the rotation imposed by the flow when the main spring is compressed $\left(\ell<\ell_{m}\right)$. Again, the opposite effect is observed for an elongated particle aligned in the shear flow direction. The leading effect, that slows down or accelerates the angular velocity, comes from the main springs to the satellite beads (flagella) and the supporting springs reduce the effect.

When the flagella plane and the shear plane are identical, each main spring exerts a constant torque during the tumbling rotation of the swimmer such that the angular velocity is almost constant on the orbit as in Fig. 3(a).

We calculated the intrinsic viscosity over a wide range of shear rates $\dot{\gamma}$ and found a shear thinning behavior as shown in Fig. 5. This is very similar to the experimentally observed shear thinning [19]. The shear thinning for an active microswimmer suspension is related to the existence of two timescales: the orbiting period $T_{b}$ of the satellite beads and the tumbling period $T$ depending on the shear rate. In the range $T / T_{b}>100$ (i.e. $\dot{\gamma} \lesssim \omega / 50$ ) one has many breast-strokes per tumbling period $T$. Therefore, the satellite motion can significantly influence the swimmers orientation thus enhancing the swimmers tumbling period. Increasing the shear rate decreases the tumbling period, the number of breast-strokes per tumbling period decreases as well as the influence of the swimming motion on the swimmers orientation. We observe a small enhancement of the intrinsic viscosity around $400-1000 H z$. This is a model related effect. At a shear rate of $5 \mathrm{~Hz}$, the ratio $T / T_{b}$ is about 150 (see table III). In the range of shear rates, $400-1000 H z$, the time scales $T$ and $T_{b}$ become comparable. The re-increase of the viscosity is due to a synchronization between the rotating beads and the tumbling and depends on the parameters of the model. This effect is probably absent for a real $C R$.

Note that the shear thinning behavior is almost vanishing for an inactive swimmer, because the satellite beads are only connected by one spring and therefore very flexible. When each satellite bead is still connected by three springs but the equilibrium length are kept constant (inactive), then the effective radius of the swimmer is enhanced compared to the inactive swimmer. A configuration of rather rigidly connected satellite beads does not correspond to the flexible flagella of a dead $C R$. However, this extension of the effective radius contributes besides the active swimming motion also to the enhanced intrinsic viscosity. However, this contribution reduces when increasing the shear rate due to the strong deformations of springs.

\section{CONCLUSION}

A three-dimensional bead spring model has been developed for Chlamydomonas Reinhardtii that takes into account the flagellar beating. The model correctly reproduces most of the swimming characteristics of this microswimmer. Using the model, we found a reversed Jeffery like orbit for the microswimmer in the shear flow. Such an altered orbit is essentially at the origin of the enhancement of suspension viscosity. We determine the intrinsic viscosity of an active and an inactive swimmer suspension using numerical simulations of the Stokesian dynamics of the three bead model within the generalized Rotne-Prager approximation. Our numerical results for the intrinsic viscosity are very similar to previous experimental measurements [19, 20] including a shear thinning behavior.

Our numerical results suggest that the significant increase of the viscosity for puller-type active microswimmer suspensions can be explained by considering the activity of an individual swimmer without a collective behavior. This effect could probably be combined with the diffusive trajectory of a $C R$ which also affects the effective viscosity [25] of dilute suspensions.

The complex angular orbits of the model swimmer and its consequences on the suspension viscosity for different orientations of the swimmer with respect of the flow direction, emphasize the importance of using a threedimensional model for such a system.

Acknowledgments.- We all acknowledge the FrenchGerman University (AUF) and the French-German Doctoral School "Living Fluids".
[1] E. Lauga and T. R. Powers, Phys. Rep. 72, 096601 (2009).
[2] D. L. Koch and G. Subramanian, Annu. Rev. Fluid Mech. 43, 637 (2011). 
[3] J. S. Guasto, R. Rusconi, and R. Stocker, Annu. Rev. Fluid Mech. 44, 373 (2012).

[4] E. Lauga, Annu. Rev. Fluid Mech. 48, 105 (2016).

[5] C. Bechinger, R. D. Leonardo, H. Löwen, C. Reichardt, G. Volpe, and G. Volpe, Rev. Mod. Phys. 88, 045006 (2016).

[6] R. Dreyfus, J. Baudry, M. L. Roper, M. Fermigier, H. A. Stone, and J. Bibette, Nature 437, 862 (2005).

[7] E. E. Keaveny and M. R. Maxey, J. Fluid Mech. 598, 293 (2008).

[8] N. Casic, N. Quintero, F. G. M. R. Alvarez-Nodarse, L. Jibuti, W. Zimmermann, and T. M. Fischer, Phys. Rev. Lett. 110, 168302 (2013).

[9] E. M. Purcell, Am. J. Phys. 45, 3 (1977).

[10] B. Kaoui, G. H. Ristow, I. Cantat, C. Misbah, and W. Zimmermann, Phys. Rev. E 77, 021903 (2008).

[11] B. M. Haines, I. S. Aranson, L. Berlyand, and D. A. Karpeev, Phys. Biol. 5, 046003 (2008).

[12] Y. Hatwalne, S. Ramaswamy, M. Rao, and R. A. Simha, Phys. Rev. Lett. 92, 118101 (2004).

[13] D. Saintillan, Exp. Mech. 50, 1275 (2010).

[14] B. M. Haines, Ph.D. thesis, The Pennsylvania State University (2011).

[15] S. Heidenreich, S. Hess, and S. H. L. Klapp, Phys. Rev. E 83, 011907 (2011).

[16] Z. Cui, Phys. Rev. E. 83, 031911 (2011).

[17] T. Ishikawa and T. J. Pedley, J. Fluid Mech. 588, 399 (2007).

[18] S. Sokolov and I. S. Aranson, Phys. Rev. Lett. 103, 148101 (2009).

[19] S. Rafai, L. Jibuti, and P. Peyla, Phys. Rev. Lett. 104, 098102 (2010).

[20] M. Mussler, S. Rafai, P. Peyla, and C. Wagner, EPL 101, 54004 (2013).

[21] J. Gachelin, G. Mino, H. Berthet, A. Lindner, A. Rousselet, and E. Clément, Phys. Rev. Lett. 110, 268103 (2013).

[22] H. M. López, J. Gachelin, C. Douarche, H. Auradou, and E. Clément, Phys. Rev. Lett. 115, 028301 (2013).

[23] W. M. Durham, J. O. Kessler, and R. Stocker, Science 323, 1067 (2009).
[24] G. B. Jeffery, Proc. R. Soc. A 102, 161 (1922).

[25] S. C. Takatori and J. F. Brady, Phys. Rev. Lett. 118, 018003 (2017).

[26] V. Mehandia and P. Nott, J. Fluid Mech. 595, 239 (2008).

[27] K. Drescher, R. E. Goldstein, N. Michel, M. Polin, and I. Tuval, Phys. Rev. Lett. 105, 168101 (2010).

[28] L. Jibuti, L. Qi, C. Misbah, W. Zimmermann, S. Rafai, and P. Peyla, Phys. Rev. E 90, 063019 (2014).

[29] B. M. Friedrich and F. Jülicher, Phys. Rev. Lett. 109, 138102 (2012).

[30] R. R. Bennett and R. Golestanian, Phys. Rev. Lett. 110, 148102 (2013).

[31] K. Polotzek and B. M. Friedrich, New. J. Phys. 15, 045005 (2013).

[32] J. Happel and H. Brenner, Low Reynolds Number Hydrodynamics (Prentice-Hall, Englewood Cliffs, 1981).

[33] E. Wajnryb, K. A. Mizerski, P. J. Zuk, and P. Szymczak, J. Fluid Mech. 731, R3 (2013).

[34] J. Rotne and S. Prager, J. Chem. Phys. 50, 4831 (1969).

[35] M. Garcia, S. Berti, P. Peyla, and S. Rafai, Phys. Rev. E (R) 83, 035301 (2011).

[36] G. W. David Stern, Elizabeth Harris, ed., The Chlamydomonas Sourcebook (Academic, 2008).

[37] Michael Reichert, PhD thesis: Hydrodynamic Interactions in Colloidal and Biological Systems, University of Konstanz, (2006).

[38] A. Einstein, Ann. Phys. (Leipzig) 19, 289 (1906).

[39] A. Einstein, Ann. Phys. (Leipzig) 34, 591 (1911).

[40] G. K. Batchelor, J. Fluid. Mech. 41, 545 (1970).

[41] L. D. Landau and E. M. Lifshitz, Course of Theoretical Physics, Vol. 6 Fluid Mechanics (Butterworth, Boston, 1987).

[42] A. S. Sangani, A. Acrivos, and P. Peyla, Phys. Fluids 23, 083302 (2013).

[43] I. M. Krieger and T. J. Dougherty, Trans. Soc. Rheol. 3, 137 (1959).

[44] D. R. Mikulencak and J. F. Morris, J. Fluid Mech. 520, 215 (2004). 